\title{
DETERMINACY OF COMPLEX ANALYTIC FOLIATION GERMS WITHOUT INTEGRATING FACTORS
}

\author{
DOMINIQUE CERVEAU AND TATSUO SUWA
}

(Communicated by Clifford J. Earle, Jr.)

\begin{abstract}
It is shown that for a codimension 1 foliation germ without formal integrating factors, the finiteness of the dimension of the space of isomorphism classes of first order unfoldings implies its local finite determinacy. An effective estimate of the order of determinacy is given for general foliation germs in dimension 2 . A simple proof for the stability of a foliation germ defined by a simple form in dimension 3 is also included.
\end{abstract}

A codim 1 foliation germ $F$ is defined by a germ of 1 -form $\omega$ satisfying the integrability condition $d \omega \wedge \omega=0$, and an unfolding of $F=(\omega)$ is a codim 1 foliation germ on an enlarged space that reduces to the original one when restricted to the original space. In $[6,8]$ it is proved that an infinitesimally versal unfolding is versal (versality theorems), and in [7] it is proved that if $F$ is infinitesimally $k$-determined, then it is locally $k$-determined. This article contains some applications of these results. Especially we give some determinacy results for foliation germs without integrating factors.

In $\S 1$ we recall definitions and results concerning the unfolding theory and determinacy of codim 1 complex analytic foliation germs. In $\S 2$ we prove that for a foliation germ $F=(\omega)$ without formal integrating factors, the finiteness of the dimension of the space $I(\omega) / J(\omega)$ of isomorphism classes of first order unfoldings implies its local finite determinacy $((2.1)$ Theorem). We also give a sufficient condition for the finite dimensionality of $I(\omega) / J(\omega)$ in terms of the singular sets of $\omega$ and of $d \omega$. The singular set of $d \omega$ is also related to the unfolding property of $F=(\omega)$. In $\S 3$ we give, as an application of the versality theorem, a simple proof of a theorem of Camacho and Lins Neto asserting that in dimension 3 , if the singular set of $d \omega$ is isolated, then $F$ is stable; i.e., every unfolding of $F$ is trivial. In $\S 4$ we give an effective estimate of the order of determinacy for general foliation germs in dimension 2 .

\section{Preliminaries}

We recall a theorem of local finite determinacy for codim 1 foliation germs proved in [7]. For this we need some language from the unfolding theory for such germs [6-9].

Received by the editors January 29, 1990.

1980 Mathematics Subject Classification (1985 Revision). Primary 32B30, 32G11; Secondary $58 \mathrm{C} 27,58 \mathrm{~F} 14$. 
Let $\mathscr{O}_{n}$ denote the ring of germs of holomorphic functions at the origin 0 in $\mathbb{C}^{n}=\left\{\left(x_{1}, \ldots, x_{n}\right)\right\}$, and let $\Omega_{n}$ and $\Theta_{n}$ denote, respectively, the $\mathscr{O}_{n}$-modules of germs of holomorphic 1 -forms and of holomorphic vector fields at 0 in $\mathbb{C}^{n}$. A codim 1 foliation germ at 0 in $\mathbb{C}^{n}$ is a rank 1 free sub- $\mathscr{O}_{n}$-module $F=(\omega)$ of $\Omega_{n}$ with a generator $\omega$ satisfying the integrability condition $d \omega \wedge \omega=0$. The germ at 0 of the analytic set $\{x \mid \omega(x)=0\}$ is denoted by $S(\omega)$ or $S(F)$ and is called the singular set of $F$. We always assume that $\operatorname{codim} S(F) \geq 2$.

An unfolding of $F=(\omega)$ is a codim 1 foliation germ $\mathscr{F}=(\tilde{\omega})$ at 0 in $\mathbb{C}^{n} \times \mathbb{C}^{m}$, for some $m$, with a generator $\tilde{\omega}$ such that $\imath^{*} \tilde{\omega}=\omega$, where $l$ denotes the embedding of $\mathbb{C}^{n}=\{x\}$ into $\mathbb{C}^{n} \times \mathbb{C}^{m}=\{(x, t)\}$ given by $l(x)=(x, 0)$. We call $\mathbb{C}^{m}$ the parameter space of $\mathscr{F}$. If we also denote by $l_{t}$ the embedding of $\mathbb{C}^{n}$ into $\mathbb{C}^{n} \times \mathbb{C}^{m}$ given by $l_{t}(x)=(x, t)$ for $t$ near 0 , we may think of $\omega_{t}=l_{t}^{*} \tilde{\omega}$ as a deformation of $\omega$. For the notions of a morphism and an $R L$-morphism, we refer to [7, (2.1) Definition] or [9, (2.1) Definition]. For a codim 1 foliation germ $F=(\omega)$, we associate the following algebraic objects [7, p. 432]; [8, p. 601]:

$$
\begin{aligned}
& I(\omega)=\left\{h \in \mathscr{O}_{n} \mid h d \omega=\eta \wedge \omega \text { for some } \eta \text { in } \Omega_{n}\right\}, \\
& J(\omega)=\left\{h \in \mathscr{O}_{n} \mid h=i_{X} \omega \text { for some } X \text { in } \Theta_{n}\right\}, \\
& K(\omega)=\left\{h \in \mathscr{O}_{n} \mid h d \omega=d h \wedge \omega\right\},
\end{aligned}
$$

where $i_{X}$ denotes the interior product with respect to the vector field $X$. Also, for a positive integer $k$, we set

$$
I^{(k)}(\omega)=\left\{h \in \mathscr{O}_{n} \mid h d \omega+(\theta-d h) \wedge \omega=0 \text { for some } \theta \text { in } \mathfrak{m}^{k-1} \Omega_{n}\right\},
$$

where $\mathfrak{m}$ denotes the maximal ideal in $\mathscr{O}_{n}$. Note that $I(\omega)$ is an ideal in $\mathscr{O}_{n}$, and $J(\omega)$ is a subideal of $I(\omega)$ [7, (2.8) Corollary]. They are independent of the choice of the generator $\omega$ of $F$. By setting $I^{(\infty)}(\omega)=K(\omega)$, which is the set of integrating factors of $\omega$, we have a decreasing sequence of $\mathbb{C}$-vector subspaces of $I(\omega)$ :

$$
I(\omega)=I^{(1)}(\omega) \supset I^{(2)}(\omega) \supset \cdots \supset I^{(k)}(\omega) \supset \cdots \supset I^{(\infty)}(\omega)=K(\omega) .
$$

If $\omega^{\prime}$ is another generator of $F$, then $\omega^{\prime}=u \omega$ for some unit $u$ in $\mathscr{O}_{n}$. The correspondence $h \mapsto u h$ gives an isomorphism of $I^{(k)}(\omega)$ onto $I^{(k)}\left(\omega^{\prime}\right)$ for each $k=1,2, \ldots, \infty$. The quotients $I(\omega) / J(\omega)$ and $I(\omega) / J(\omega)+K(\omega)$ are interpreted, respectively, as the sets of isomorphism classes and of $R L$ isomorphism classes of first order unfoldings of $F=(\omega)$ [8, (1.4) Proposition].

For a germ $g$ in $\mathscr{O}_{n}$, we denote by $j^{k} g$ the $k$-jet of $g$, and for a germ $\theta=\sum_{i=1}^{n} g_{i} d x_{i}$ in $\Omega_{n}$, we define the $k$-jet $j^{k} \theta$ of $\theta$ by

$$
j^{k} \theta=\sum_{i=1}^{n} j^{k-1} g_{i} \cdot d x_{i} .
$$

(1.1) Definition. An unfolding $\mathscr{F}$ of a codim 1 foliation germ $F=(\omega)$ with parameter space $\mathbb{C}^{m}=\{t\}$ is $k$-trivial if it has a generator $\tilde{\omega}$ such that $\imath^{*} \tilde{\omega}=\omega$ and that $j^{k} \omega_{t}=j^{k} \omega$ for all $t$ near 0 in $\mathbb{C}^{m}$. 
We say that two germs $\omega$ and $\omega^{\prime}$ in $\Omega_{n}$ are holomophically equivalent and write $\omega \sim \omega^{\prime}$ if there exist a germ at 0 of biholomorphic map $\varphi$ of $\mathbb{C}^{n}$ into itself with $\varphi(0)=0$ and a unit $u$ in $\mathscr{O}_{n}$ such that $\omega^{\prime}=u \varphi^{*} \omega$. If $\omega$ is integrable and if $\omega^{\prime} \sim \omega, \omega^{\prime}$ is also integrable, and $\omega$ and $\omega^{\prime}$ define isomorphic foliations.

(1.2) Definition. A codim 1 foliation germ $F(\omega)$ is locally $k$-determined if for every $k$-trivial unfolding $\mathscr{F}=(\tilde{\omega})$ of $F$, we have $\omega_{t} \sim \omega$ for all $t$ near 0 .

(1.3) Definition. A codim 1 foliation germ $F=(\omega)$ is infinitesimally $k$ determined if

$$
I^{(k+1)}(\omega) \subset \mathfrak{m} J(\omega)+K(\omega) .
$$

For the geometric meaning of the above condition, see [7, p. 437]. We quote the following:

(1.4) Theorem [7]. Let $F=(\omega)$ be a codim 1 foliation germ with

$$
\operatorname{dim} K(\omega) / \mathfrak{m} J(\omega) \cap K(\omega)<+\infty .
$$

If $F$ is infinitesimally $k$-determined, then it is locally $k$-determined.

(1.5) Remark. Actually, in [7] it is shown that under the assumption $(*)$, if $F$ is infinitesimally $k$-determined, then every $k$-trivial (one parameter) undolfing of $F$ is (strongly) $R L$-trivial. Moreover, when $K(\omega)=0$, every $k$-trivial (one parameter) unfolding of $F$ is (strongly) trivial.

\section{DETERMINACY OF FOLIATION GERMS WITHOUT FORMAL INTEGRATING FACTORS}

A formal integrating factor of an integrable 1 -form $\omega$ is a formal power series $\hat{h}$ in $x=\left(x_{1}, \ldots, x_{n}\right)$ such that $\hat{h} d \omega=d \hat{h} \wedge \omega$. We say that a codim 1 foliation germ $F=(\omega)$ is locally finitely determined if it is locally $k$-determined for some $k$.

(2.1) Theorem. Let $F=(\omega)$ be a codim 1 foliation germ at 0 in $\mathbb{C}^{n}$ without (nonzero) formal integrating factors. If the $\mathbb{C}$-vector space $I(\omega) / J(\omega)$ is finite dimensional, then $F$ is locally finitely determined.

To prove this, we first prove the following:

(2.2) Lemma. Let $F=(\omega)$ be a codim 1 foliation germ without formal integrating factors. For every positive integer $k$, there exists another $k^{\prime}$ such that

$$
I^{\left(k^{\prime}\right)}(\omega) \subset \mathfrak{m}^{k} \cap I(\omega) .
$$

Proof. If $I^{(k)}(\omega)=\{0\}$ for some $k$, then there is nothing to prove. Then we assume that $I^{(k)}(\omega) \neq\{0\}$ for all $k$. For each $k$, we set

$$
n(k)=\min \left\{\nu(h) \mid h \in I^{(k)}(\omega)\right\},
$$


where $\nu(h)$ denotes the order of $h$. We have an increasing sequence $\{n(k)\}$ of nonnegative integers. For our purpose, it suffices to show that

$$
\lim _{k \rightarrow \infty} n(k)=+\infty \text {. }
$$

Suppose the sequence is bounded, and let $n_{0}$ be an integer such that $n(k) \leq n_{0}$ for all $k$. Then $j^{n_{0}} I^{(k)}(\omega) \neq\{0\}$ for all $k$. We have a decreasing sequence of finite-dimensional vector spaces

$$
\cdots \supset j^{n_{0}} I^{(k)}(\omega) \supset j^{n_{0}} I^{(k+1)}(\omega) \supset \cdots \supsetneqq\{0\} .
$$

Thus, there exists $k_{0}$ such that for all $k \geq k_{0}$,

$$
j^{n_{0}} I^{(k)}(\omega)=j^{n_{0}} I^{\left(k_{0}\right)}(\omega) .
$$

We fix a germ $H$ in $I^{\left(k_{0}\right)}(\omega)$ with $j^{n_{0}} H \neq 0$. Then for all $k$, there exists $h$ in $I^{(k)}(\omega)$ such that $j^{n_{0}} h=j^{n_{0}} H$. We claim that there exists a nonzero formal power series $\hat{h}$ in $x$ such that for all $n$, we have

$(2.3)_{n} \quad$ for all $k$, there exist $h$ in $I^{(k)}(\omega)$ with $j^{n} h=j^{n} \hat{h}$.

We determine the $n$-jet of $\hat{h}$ for every $n$ by induction. If we let the $n_{0}$-jet of $\hat{h}$ be equal to $j^{n_{0}} H$, then we have $(2.3)_{n}$ for $n \leq n_{0}$. Suppose we have determined the $n$-jet of $\hat{h}$ so that $(2.3)_{n}$ is satisfied. We set

$$
I^{(k)}(\omega)_{n}=\left\{h \in I^{(k)}(\omega) \mid j^{n} h=j^{n} \hat{h}\right\},
$$

which is a (nonempty) affine subspace of $I(\omega)$. We have a decreasing sequence of finite-dimensional affine spaces:

$$
\cdots \supset j^{n+1} I^{(k)}(\omega)_{n} \supset j^{n+1} I^{(k+1)}(\omega)_{n} \supset \cdots .
$$

Thus, there exists $k_{1}$ such that for all $k \geq k_{1}$,

$$
j^{n+1} I^{(k)}(\omega)_{n}=j^{n+1} I^{\left(k_{1}\right)}(\omega)_{n} .
$$

Hence there exists a homogeneous polynomial $h_{n+1}$ of degree $n+1$ such that $j^{n} \hat{h}+h_{n+1}$ is in $j^{n+1} I^{(k)}(\omega)$ for all $k$. If we let the $n+1$ jet of $\hat{h}$ be equal to $j^{n} \hat{h}+h_{n+1}$, then we have $(2.3)_{n+1}$.

Clearly the formal power series $\hat{h}$ thus constructed is a nonzero formal integrating factor of $\omega$.

Proof of (2.1) Theorem. By (1.4) Theorem, it suffices to show that $I^{(k)}(\omega) \subset$ $\mathfrak{m} J(\omega)$ for some $k$. First by the finite dimensionality of $I(\omega) / J(\omega)$, we have

$$
\mathfrak{m}^{k} I(\omega) \subset \mathfrak{m} J(\omega)
$$

for some $k$. Second by Artin-Rees theorem (e.g. [5]), we have

$$
\mathfrak{m}^{k^{\prime}} \cap I(\omega) \subset \mathfrak{m}^{k} \cdot I(\omega)
$$


for some $k^{\prime}$. Finally by (2.2) Lemma, we have

for some $k^{\prime \prime}$. Q.E.D.

$$
I^{\left(k^{\prime \prime}\right)}(\omega) \subset \mathfrak{m}^{k^{\prime}} \cap I(\omega)
$$

When $n=2$, by the assumption $\operatorname{codim} S(\omega) \geq 2, \mathscr{O}_{2} / J(\omega)$ is always finite dimensional. Thus we have:

(2.4) Corollary. Every codim 1 foliation germ at 0 in $\mathbb{C}^{2}$ without formal integrating factors is locally finitely determined.

(2.5) Remarks. $1^{\circ}$. Let $F=(\omega)$ be a codim 1 foliation germ without formal integrating factors. The above arguments show that if $I(\omega) / J(\omega)$ is finite dimensional, then $F$ is infinitesimally finitely determined. The converse is also true, since $K(\omega)=\{0\}$ in this case (see [7, (4.3) Remark]).

$2^{\circ}$. Let $F=(\omega)$ be a codim 1 foliation germ at 0 in $\mathbb{C}^{2}$. If $\omega$ is "nonreal" (cf. [3, p. 136]) and if $K(\omega)=0$, then $\omega$ has no nonzero formal integrating factor [3, p. 136, Proposition 3.2]. Note that for general $\omega$, we have $K(\omega)=0$ ((4.6) Remark).

$3^{\circ}$. Let $F=(\omega)$ be a codim 1 foliation germ at 0 in $\mathbb{C}^{n}$. If $K(\omega) \neq 0$, then $\omega$ admits a multiform first integral [3, Théorème d'intégration].

$4^{\circ}$. As for the determinacy problem for multiform functions see [3, Sixième partie; $7, \S 5]$.

Here is a case when we have the finite dimensionality of $I(\omega) / J(\omega)$. Let $F=(\omega)$ be a codim 1 foliation germ. If we let $S(d \omega)$ be (the germ at 0 of) the zero set of $d \omega$, we easily see that $S(\omega) \cap S(d \omega)$ does not depend on the choice of the generator $\omega$ of $F$.

(2.6) Proposition. Let $F=(\omega)$ be a codim 1 foliation germ. If $S(\omega) \cap$ $S(d \omega) \subset\{0\}$, then the vector space $I(\omega) / J(\omega)$ is finite dimensional.

Proof. The finite dimensionality of $I(\omega) / J(\omega)$ is equivalent to saying that the support of the coherent sheaf $I(\omega) / J(\omega)$ is contained in $\{0\}$. Take a point $x \neq 0$ near 0 . By the assumption we have either $\omega(x) \neq 0$ or $d \omega(x) \neq 0$. Suppose $\omega(x) \neq 0$. Then $J(\omega)_{x}=I(\omega)_{x}=\mathscr{O}_{x}$. Suppose $d \omega(x) \neq 0$. For a germ $h$ in $I(\omega)_{x}$, we have $h d \omega=\eta \wedge \omega$ for some $\eta$ in $\Omega_{x}$. Since $d \omega(x) \neq 0$, $h$ is in $J(\omega)_{x}$.

\section{STABILITY OF SIMPLE FORMS IN $\mathbb{C}^{3}$}

Let $\omega$ be a germ of integrable 1 -form. As we noted in the previous section, the size of the zero set of $d \omega$ seems to have some effect on the determinacy of $\omega$. It is also related to the unfolding property of $\omega$. In Camacho-Lins Neto [1], a germ $\omega$ at 0 in $\mathbb{C}^{3}$ is called simple if $S(d \omega) \subset\{0\}$ and it is proved that a simple form $\omega$ is stable; i.e., every unfolding of $\omega$ is trivial [1 Theorem 4]. In this section, we give a simple proof of this result as a direct application of the versality theorem in [6]. 
(3.1) Theorem. Let $\omega$ be a germ of integrable 1-form at 0 in $\mathbb{C}^{3}$. If $S(d \omega) \subset$ $\{0\}$, then $I(\omega) / J(\omega)=0$. Thus every unfolding of the foliation $F=(\omega)$ is trivial.

Proof. First, we proceed as in the proof of Theorem 1 in [1]. Thus, denoting by $(x, y, z)$ coordinates of $\mathbb{C}^{3}$, we let $X$ be the vector field germ such that

$$
d \omega=i_{X}(d x \wedge d y \wedge d z) .
$$

From the integrability of $\omega$, we easily see that $i_{X} \omega=0$. Hence by the assumption on $S(d \omega)$, we may write $\omega=i_{X} \tau$ for some 2-form $\tau$. If we let $Y$ be the vector field such that $\tau=i_{Y}(d z \wedge d y \wedge d z)$, we get

$$
\omega=i_{X} i_{Y}(d x \wedge d y \wedge d z) \text {. }
$$

Next, we let $F=(\omega)$ be the $\mathscr{O}$-module generated by $\omega$, and let $F^{a}$ be the annihilator of $F$ in $\Theta$ :

$$
F^{a}=\left\{Z \in \Theta \mid i_{Z} \omega=0\right\} .
$$

Obviously the vector fields $X$ and $Y$ are in $F^{a}$ and generate $F^{a}$ outside of $S(\omega)$. Since codim $S(\omega) \geq 2$, they also generate $F^{a}$ across $S(\omega)$.

Now we take a germ $h$ in $I(\omega)$. Then $h d \omega=\eta \wedge \omega$ for some $\eta$ in $\Omega$. By assumption, for any point $x(\neq 0)$ near $0, d \omega(x) \neq 0$. Hence the germ $h_{x}$ of $h$ at $x$ is in $J(\omega)_{x}$. We take a small Stein open neighborhood $U$ of 0 in $\mathbb{C}^{3}$. There is a covering $\mathscr{U}=\left\{U_{\alpha}\right\}$ of $U \backslash\{0\}$ by small open sets $U_{\alpha}$ such that on each $U_{\alpha}$, we may write

$$
h=i_{X_{\alpha}} \omega
$$

for some vector field $X_{\alpha}$ on $U_{\alpha}$. In the intersection $U_{\alpha} \cap U_{\beta}$, we have $i_{X_{\alpha-X_{\beta}}}(\omega)=0$. Hence $X_{\alpha}-X_{\beta}$ is in $F^{a}$ and we may write

$$
X_{\alpha}-X_{\beta}=f_{\alpha \beta} X+g_{\alpha \beta} Y
$$

for holomorphic functions $f_{\alpha \beta}$ and $g_{\alpha \beta}$ on $U_{\alpha} \cap U_{\beta}$. We have the cocycles $\left\{f_{\alpha \beta}\right\}$ and $\left\{g_{\alpha \beta}\right\}$, which determine cohomology classes in $H^{1}(\mathscr{U}, \mathscr{O})=$ $H^{1}(U \backslash\{0\}, \mathscr{O})$. Since this cohomology group vanishes, we may write

$$
f_{\alpha \beta}=f_{\beta}-f_{\alpha} \text { and } g_{\alpha \beta}=g_{\beta}-g_{\alpha}
$$

for holomorphic functions $f_{\alpha}$ and $g_{\alpha}$ on $U_{\alpha}$. Thus we have a vector field $Z$ on $U \backslash\{0\}$, which is given by

$$
Z=X_{\alpha}+f_{\alpha} X+g_{\alpha} Y \text { on } U_{\alpha}
$$

We have

$$
h=i_{Z} \omega
$$

on $U \backslash\{0\}$. The vector field $Z$ extends across 0 , and we have (3.2) in $\mathscr{O}$. Hence $h$ is in $J(\omega)$, and we proved that $I(\omega) / J(\omega)=0$. By [6, (4.1) Corollary], every unfolding of $F=(\omega)$ is trivial. Q.E.D. 


\section{EFFECTIVE ESTIMATE OF THE ORDER OF DETERMINACY}

In this section, we consider foliation germs at 0 in $\mathbb{C}^{2}=\{(x, y)\}$. We denote by $\Omega_{k}^{p}$ the vector space of homogeneous $p$-forms of degree $k$ on $\mathbb{C}^{2}$. The space $\Omega_{k}^{0}$ of homogeneous polynomials of degree $k$ in $(x, y)$ is especially denoted by $\mathscr{O}_{k}$. Thus, we may write $\Omega_{k}^{1}=\mathscr{O}_{k-1} d x \oplus \mathscr{O}_{k-1} d y$ and $\Omega_{k}^{2}=$ $\mathscr{O}_{k-2} d x \wedge d y$. Note that our definition of the degree of a form is different from the conventional one.

Let $\omega_{k_{0}}$ be an element in $\Omega_{k_{0}}^{1}$. Denoting by $R$ the radial vector field:

$$
R=x \partial / \partial x+y \partial / \partial y,
$$

we set

$$
P_{k_{0}}=i_{R} \omega_{k_{0}}
$$

Hereafter we only consider the case $P_{k_{0}} \neq 0$; i.e., $\omega_{k_{0}}$ is nondicritic. We easily see that $P_{k_{0}}$ is an integrating factor of $\omega_{k_{0}}$.

(4.1) Definition ([3, p. 134]; Cf. also [4, 2]). We say that $\omega_{k_{0}}$ is generic if

(a) $S\left(\omega_{k_{0}}\right) \subset\{0\}$.

(b) $P_{k_{0}}$ is reduced.

(c) $\omega_{k_{0}}$ does not admit meromorphic first integrals, i.e., there are no nonconstant meromorphic function germs $\varphi$ with $d \varphi \wedge \omega_{k_{0}}=0$.

Suppose $\omega_{k_{0}}$ is generic. Since the quotient of two integrating factors of $\omega_{k_{0}}$ is a meromorphic first integral of $\omega_{k_{0}}$, the condition (c) implies that

$$
K\left(\omega_{k_{0}}\right)=\mathbb{C} P_{k_{0}} .
$$

We set

$V\left(\omega_{k_{0}}\right)=\left\{\theta \in \Omega_{k_{0}+1}^{1} \mid P_{k_{0}} d \theta-d P_{k_{0}} \wedge \theta=d f \wedge \omega_{k_{0}}-f d \omega_{k_{0}}\right.$ for some $f$ in $\left.\mathscr{O}_{k_{0}+1}\right\}$.

(4.3) Lemma. The set $V\left(\omega_{k_{0}}\right)$ is a subvector space of $\Omega_{k_{0}+1}^{1}$ of codimension at least $2 k_{0}-\min \left\{2 k_{0}, k_{0}+2\right\}$.

Proof. We consider two linear maps

$$
\lambda: \Omega_{k_{0}+1}^{1} \rightarrow \Omega_{2 k_{0}+1}^{2} \text { and } \mu: \mathscr{O}_{k_{0}+1} \rightarrow \Omega_{2 k_{0}+1}^{2}
$$

defined by $\lambda(\theta)=P_{k_{0}} d \theta-d P_{k_{0}} \wedge \theta$ for $\theta$ in $\Omega_{k_{0}+1}^{1}$ and by $\mu(f)=d f \wedge \omega_{k_{0}}-$ $f d \omega_{k_{0}}$ for $f$ in $\mathscr{O}_{k_{0}+1}$. By [3, Théorème d'intégration], we see that

$$
\text { Ker } \lambda=\left\{\theta \in \Omega_{k_{0}+1}^{1} \mid \theta=P_{k_{0}} d L \text { for some } L \text { in } \mathscr{O}_{1}\right\} .
$$

Hence $\operatorname{rank} \lambda=2\left(k_{0}+1\right)-2=2 k_{0}$. On the other hand, by (4.2), Ker $\mu=0$. Hence rank $\mu=k_{0}+2$. Thus we have

$$
\begin{aligned}
\operatorname{dim} V\left(\omega_{k_{0}}\right) & =\operatorname{dim} \operatorname{Ker} \lambda+\operatorname{dim}(\operatorname{Im} \lambda \cap \operatorname{Im} \mu) \\
& \leq 2+\min \left\{2 k_{0}, k_{0}+2\right\} .
\end{aligned}
$$


(4.4) Theorem. Let $\omega$ be a germ of integrable 1-form at 0 in $\mathbb{C}^{2}$ and let $\omega_{k_{0}}$ be the first nonzero jet of $\omega$. Further, we let $\omega_{k_{0}+1}=j^{k_{0}+1} \omega-\omega_{k_{0}}$. Suppose $k_{0} \geq 3, \omega_{k_{0}}$ is generic and $\omega_{k_{0}+1}$ is not in $V\left(\omega_{k_{0}}\right)$. Then

$$
I^{(k)}(\omega)=\mathfrak{m}^{k} \cap I(\omega)
$$

for $k>k_{0}+1$.

Proof. For a germ of holomorphic function $h$ and a germ of holomorphic 1form $\theta$, we set $h_{k}=j^{k} h-j^{k-1} h$ and $\theta_{k}=j^{k} \theta-j^{k-1} \theta$. In what follows, we fix $f$ with $k>k_{0}+1$. If $h$ is a germ in $I^{(k)}(\omega)$, we have

$$
h d \omega+(\theta-d h) \wedge \omega=0
$$

for some $\theta$ with $j^{k-1} \theta=0$. From this, we easily see that

$$
j^{k_{0}-1}=0 \text { and } h_{k_{0}} \in K\left(\omega_{k_{0}}\right) .
$$

Thus by (4.2), we may write $h_{k_{0}}=c P_{k_{0}}$ for some $c$ in $\mathbb{C}$. Comparing the terms of degree $2 k_{0}+1$ in (4.5), we get

$$
c\left(P_{k_{0}} d \omega_{k_{0}+1}-d P_{k_{0}} \wedge \omega_{k_{0}+1}\right)=d h_{k_{0}+1} \wedge \omega_{k_{0}}-h_{k_{0}+1} d \omega_{k_{0}} .
$$

Since $\omega_{k_{0}+1}$ is not in $V\left(\omega_{k_{0}}\right)$, we have $c=0$. Thus $j^{k_{0}} h=0$. Then, by (4.5) and (4.2), we see that

$$
j^{k_{0}+1} h=\cdots=j^{k-1} h=0 .
$$

Hence we have $I^{(k)}(\omega) \subset \mathfrak{m}^{k} \cap I(\omega)$. Conversely, let $h$ be a germ in $\mathfrak{m}^{k} \cap I(\omega)$. We have the identity (4.5) for some $\theta$ in $\Omega$. By condition (a) in (4.1), if $\eta \wedge \omega_{k_{0}}=0$ for $\eta$ in $\Omega$, then $\eta=g \omega_{k_{0}}$ for some $g$ in $\mathscr{O}$. Thus from (4.5), we see that

$$
j^{k_{0}-1} \theta=0 .
$$

Comparing the terms of degrees from $2 k_{0}$ to $k_{0}+k-1$, we see that for each $l$ with $0 \leq l \leq k-k_{0}-1$, there is an element $g_{l}$ in $\mathscr{O}_{l}$ such that

$$
\theta_{l}=\sum_{l^{\prime}=k_{0}}^{l} g_{l-l^{\prime}} \omega_{l^{\prime}}
$$

for $l$ with $k_{0} \leq l \leq k-1$. If we set $g=\sum_{l=0}^{k-k_{0}-1} g_{l}$ and $\theta^{\prime}=\theta-g \omega$, then we have

$$
h d \omega+\left(\theta^{\prime}-d h\right) \wedge \omega=0 \text { and } j^{k-1} \theta^{\prime}=0 .
$$

Hence $h$ is in $I^{(k)}(\omega)$.

(4.6) Remark. The above theorem holds also for $k=\infty$; i.e., for a germ $\omega$ as in (4.4) Theorem, $K(\omega)=0$. We also have $K\left(j^{k} \omega\right)=0$ for $k>k_{0}$. 
(4.7) Corollary. Let $\omega$ be as in (4.4) Theorem. If

$$
\mathfrak{m}^{k+1} \cap I(\omega) \subset \mathfrak{m} J(\omega)
$$

for some $k>k_{0}$, then $F=(\omega)$ is locally $k$-determined.

(4.8) Remark. By the Artin-Rees theorem, there is always $k$ for which the condition in (4.7) is satisfied.

(4.9) Example. Let $\omega$ be as in (4.4) Theorem and assume $k_{0}=3$. Then we easily see that $\mathfrak{m}^{3} \subset J\left(\omega_{s}\right)$. On the other hand, by the Nakayama lemma, we have $J(\omega)=J\left(\omega_{3}\right)$. Hence

$$
\mathfrak{m}^{4+1} \subset \mathfrak{m}^{4} \subset \mathfrak{m} J(\omega) .
$$

Thus $F=(\omega)$ is locally 4-determined.

\section{ACKNOWLEDGMENT}

Part of this work was done while the second author was visiting Universite de Dijon and Universite de Rennes. He would like to express his gratitude for their hospitality.

\section{REFERENCES}

1. C. Camacho and A. Lins Neto, The topology of integrable differential forms near a singularity, Publ. Math. Inst. Hautes Études Sci. 55 (1982), 5-35.

2. D. Cerveau, Espaces de modules de certaines equations différentielles du premier ordre, preprint.

3. D. Cerveau et J.-F. Mattei, Formes intégrables holomorphes singulières, Astérisque 97(1982).

4. D. Cerveau et P. Sad, Problèmes de modules pour les formes différentielles singulières dans le plan complexe, Comment. Math. Helv. 61 (1986), 222-253.

5. H. Matsumura, Commutative algebra, W. A. Benjamin, New York, 1970.

6. T. Suwa, $A$ theorem of versality for unfoldings of complex analytic foliation singularities, Invent. Math. 65 (1981), 29-48.

7. _ Determinancy of analytic foliation germs, Adv. Stud. Pure Math. vol. 5; Foliations (I. Tamura, ed.), Kinokuniya and North-Holland, 1985, pp. 427-460.

8. _ The versality theorem for RL-morphisms of foliation unfoldings, Adv. Stud. Pure Math. vol. 8; Complex analytic singularities (T. Suwa and P. Wagreich, eds.), Kinokuniya and North-Holland, Amsterdam, 1986, pp. 599-631.

9. _ A factorization theorem for unfoldings of analytic functions, Proc. Amer. Math. Soc. 104 (1988), 131-134.

U.E.R. Mathématiques et InformatiQue, Université de Rennes I, Campus de Beaulieu, 35042 Rennes Cedex France

Department of Mathematics, Hokkaido University, Sapporo 060 Japan 